Research Article

\title{
Biomarkers and Mechanism Analysis for Polygoni Multiflori Radix Preparata-Induced Liver Injury by UHPLC-Q-TOF-MS-Based Metabolomics
}

\author{
Liming Wang, ${ }^{1}$ Zhida Wang, ${ }^{2}$ Yanchao Xing, ${ }^{1}$ Erwei Liu, ${ }^{1}$ Xiumei Gao, ${ }^{1}$ Linlin Wang $\mathbb{D},{ }^{3}$ \\ and Zhifei Fu (iD) \\ ${ }^{1}$ State Key Laboratory of Component-based Chinese Medicine, Tianjin University of Traditional Chinese Medicine, \\ 10 Poyanghu Road, Jinghai, Tianjin 301617, China \\ ${ }^{2}$ NHC Key Laboratory of Hormones and Development, Tianjin Key Laboratory of Metabolic Diseases, \\ Chu Hsien-I Memorial Hospital \& Tianjin Institute of Endocrinology, Tianjin Medical University, Tianjin 300070, China \\ ${ }^{3}$ Second Affiliated Hospital of Tianjin University of Traditional Chinese Medicine, Tianjin 300250, China \\ Correspondence should be addressed to Linlin Wang; lynnwlin@yeah.net and Zhifei Fu; fuzhifei@tjutcm.edu.cn
}

Received 2 September 2021; Accepted 12 October 2021; Published 23 November 2021

Academic Editor: Li-Ping Kang

Copyright $\odot 2021$ Liming Wang et al. This is an open access article distributed under the Creative Commons Attribution License, which permits unrestricted use, distribution, and reproduction in any medium, provided the original work is properly cited.

\begin{abstract}
Background. Polygonum Multiflorum Radix Preparata (PMP), prepared from Polygonum multiflorum Thunb. (PM), is traditionally valued for its liver and kidney-tonifying effects. However, the previous studies showed that PMP was hepatotoxic, which limited its clinical use. Unfortunately, the potential hepatotoxic ingredients and the molecular mechanism are still uncertain. Objective. The aim of this study was to find out potential biomarkers of hepatotoxicity using metabolomics profile. Materials and Methods. 60\% ethanol extract of PMP (PMPE) was prepared. Subsequently, an untargeted metabolomics technology in combination with ROC curve analysis method was applied to investigate the alteration of plasma metabolites in rats after oral administration of PMPE ( $40 \mathrm{~g} / \mathrm{kg} / \mathrm{d})$ for 28 days. Results. Compared to the control group, the significant difference in metabolic profiling was observed in the PMPE-induced liver injury group, and sixteen highly specific biomarkers were identified. These metabolites were mainly enriched into bile acids, lipids, and energy metabolisms, indicating that PMPE-induced liver injury could be related to cholestasis and dysregulated lipid metabolism. Conclusions. This study is contributed to understand the potential pathogenesis of PMP-induced liver injury. The metabonomic method may be a valuable tool for the clinical diagnosis of PMPinduced liver injury.
\end{abstract}

\section{Introduction}

Polygoni Multiflori (PM, Heshouwu in Chinese), derived from the roots of Polygonum multiflorum Thunb., is a widely used Chinese medicinal material in clinic. Raw PM (RPM) has the effects of detoxicating, eliminating carbuncle, cutting off malaria, loosening bowel, and relieving constipation. A few of ancient books of traditional Chinese medicine, such as "ben cao hui yan," recorded that RPM is poisonous [1]. To reduce toxicity and enhance tonic effect, RPM was usually processed by black bean sauce or by nine cycles of steaming and sun drying to obtain the processed PM (PMP) [2]. PMP demonstrates the functions of nourishing the liver, kidney, and blood, strengthening the muscles and bones, and blackens hair [3-6]. Interestingly, RMP steamed with black soybeans for nine cycles was safe. However, modern processing technologies, usually steamed with the black soybean juice once, still exhibit obvious hepatotoxicity $[7,8]$. PMPrelated liver injury has been reported in many countries and regions, such as South Korea, Japan, and UK [9]. Recently, the National Medical Products Administration in China has also warned of the risk of liver injury from the extracts of RPM and PMP [10]. Unfortunately, although extensive experiments have been performed in recent years, the 
potential toxic mechanisms that cause liver injury remain unclear. Therefore, it is particularly urgent and important to establish a method for the early warning of liver injury of PMP.

Metabolome is located downstream of gene regulation and protein network and could provide terminal information of biology. Metabolomics is the study of endogenous metabolite alterations associated with maintaining the normal function and development of organisms after the stimulation or disturbance of biological systems [11]. It has been widely applied in various fields of life sciences and represents a powerful tool for interpreting life phenomena and exploring mechanisms of disease [12]. Chromatography combined with mass spectrometry (such as LC-MS and GCMS) and nuclear magnetic resonance (NMR) spectroscopy is widely applied in metabolomics studies, and LC-MS is the most popular used methodology in untargeted metabolomics research. Ultrahigh performance liquid chromatography-mass spectrometry technology provides a rapid, concise, and high-throughput approach for the analysis of biological fluids or tissues [13]. Combined with multivariate statistical analysis methods, it provides an effective method for the discovery of metabolic pathways. The targeted and untargeted methods in MS-based metabolomics were used to detect changes in the processing technologies of Polygoni Multiflori Radix [14]. In conclusion, metabolomics has been extensively used for the discovery of biomarkers and the research on the metabolic mechanism [15].

The hepatotoxicity components of PMP have always been the subject of controversy. In our previous study [16], we performed a comparative study on the chemical components in the aqueous extract and $60 \%$ ethanol extract of PMP (PMPE). The results showed that the different components were attributed to anthraquinones, such as emodin, rhein, and physcion. Furthermore, the pharmacokinetics characters of these main components in rats were studied. The $t_{1 / 2(\mathrm{~h})}$ of those components of the PMPE was longer compared to the aqueous extract of PMP and increased with the increasing of dosage of PMPE. Anthraquinone components (such as emodin) which has higher content in PMPE are regarded as potential liver damage components [16]. Based on our previous research and literature review, the PMPE showed a degree of hepatotoxicity $[4,16,17]$; therefore, we selected PMPE to investigate the components and mechanism of potential liver injury of PMP. The study aimed to further explore the potential hepatotoxicity mechanism of PMPE using the LC-MS-based metabolomics method. The reliability of discriminated metabolite was further screened by receiver operating characteristic (ROC) curve analysis, and the related metabolic pathways were clarified. Combined with biochemical indicators, our work could further clarify the potential mechanism of PMPEinduced liver injury.

\section{Materials and Methods}

2.1. Chemicals and Reagents. HPLC grade methanol (ME) and acetonitrile (ACN) were provided by Thermo Fisher Scientific Co., Ltd. (Pittsburgh, PA, USA). Ultrapure water was obtained from Watsons Food\&Beverage Co., Ltd. (Guangdong, China). PMP was purchased from Anguo Shengshan Pharmaceutical Co., Ltd. (Hebei, China).

2.2. Sample Preparation. PMP $(10 \mathrm{~kg})$ was extracted with $60 \%$ ethanol $(\mathrm{v} / \mathrm{v})$ to yield $1.7 \mathrm{~kg}$ extract (PMPE), stored at $4^{\circ} \mathrm{C}$ for further use. The main constituents in PMPE were profiled and reported by our previous research [16].

2.3. Animal and Experiment Design. Sprague Dawley (SD) rats (6-8 weeks) were purchased from HFK Bioscience Co., Ltd. (Beijing, China). All animals were kept under the same breeding room conditions with a temperature between 20 and $25^{\circ} \mathrm{C}$ and given standard laboratory water and food. The procedures involving animals and their care were conformed to the Guiding Principles for the Care and Use of Laboratory Animals of China. The rats were kept in the breeding room for 7 days and then randomly divided into different groups ( $n=7$ for each group).

Animals were randomly divided into the blank control group (BC, equivalent physiological saline) and PMPE treatment group; based on our previous studies [16], the dosage was set at $40 \mathrm{~g} / \mathrm{kg}$ per day (amount of crude drug weight of rat/day). The clinical syndromes of animals were observed every day. The animal's body weight was monitored once a week after intragastric administration.

Rats were fasted for 12 hours after oral administration of PMPE for 28 days, and blood sample of rat was collected from the abdominal aorta. One part with no anticoagulant samples remained for 2 hours, $3500 \times \mathrm{g}$ was centrifuged for $10 \mathrm{~min}$, and then serum was obtained for analysis of the serum biochemical index. The remaining serum with anticoagulant was centrifuged under $3000 \times \mathrm{g}$ for $10 \mathrm{~min}$, and finally, the plasma was obtained and frozen in the refrigerator at $-80^{\circ} \mathrm{C}$ for further analysis. The liver of each rat was taken for histological examination, and the organ index was calculated.

2.4. Serum Biochemical Analysis. The serum biochemical indexes, including alanine aminotransferase (ALT), aspartate aminotransferase (AST), total bile acid (TBA), alkaline phosphatase (ALP), total bilirubin (T-BIL), and triglyceride (TG), were measured dynamically using a Hitachi 7020 automatic biochemistry analyzer (Tokyo, Japan).

2.5. Liver Histopathology Assessment. The liver samples were fixed with $10 \%$ neutral formalin for $48-72 \mathrm{~h}$, embedded in paraffin after fixation, continuously sectioned at a thickness of $5 \mu \mathrm{m}$, stained with hematoxylin and eosin (H\&E), and evaluated using a microscope.

2.6. Plasma Preparation and UHPLC/Q-TOF-MS Analysis. An amount of $100 \mu \mathrm{L}$ of plasma was added into $400 \mu \mathrm{L}$ of acetonitrile to precipitate protein. All the samples were vortexed for $5 \mathrm{~min}$ and centrifuged at $14000 \times \mathrm{g}$ for $10 \mathrm{~min}$ under $4^{\circ} \mathrm{C}$. The supernatant was transferred into $1.5 \mathrm{~mL}$ tube 
and dried under a nitrogen stream. All samples were redissolved in $100 \mu \mathrm{L}$ of methanol-water $(80: 20$, v/v). Each reconstituted sample was centrifuged at $14000 \times \mathrm{g}$ for $10 \mathrm{~min}$ under $4^{\circ} \mathrm{C}$, and then the supernatant was transferred into sample vials for analysis using UHPLC/Q-TOF-MS. The quality control (QC) samples were prepared by adding equal amounts of all analytes. During the research process, QC was carried out every five samples to verify the stability and accuracy of the system.

LC analysis was conducted on an Agilent 1290 UHPLC system equipped with an ACQUITY UPLC HSS $\mathrm{T}_{3}$ column $(2.1 \times 100 \mathrm{~mm}, 1.8 \mu \mathrm{m}$, Waters, Ireland $)$ under the temperature of $35^{\circ} \mathrm{C}$. The mobile phase consisted of $0.1 \%$ formic acid in water (A) and acetonitrile (B). The gradient of elution conditions was as follows: $0-13 \mathrm{~min}, 3-100 \% \mathrm{~B}, 13-17 \mathrm{~min}$, $100 \% \mathrm{~B}$. The flow rate was $0.4 \mathrm{~mL} \cdot \mathrm{min}^{-1}$, and the injection volume was $2 \mu \mathrm{L}$.

The mass spectrometry analysis was performed on an Agilent 6520 Accurate-Mass Q-TOF/MS system. The conditions of the ESI source were drying gas flow rate, $8.0 \mathrm{~L} \cdot \mathrm{min}^{-1}$; drying gas temperature, $350^{\circ} \mathrm{C}$; nebulizer, $30 \mathrm{psi}$; capillary voltage (Vcap), $4000 \mathrm{~V}$ in positive ion mode, and $3500 \mathrm{~V}$ in negative ion mode; fragmentor voltage, $150 \mathrm{~V}$ and $175 \mathrm{~V}$ in positive and negative ion modes, respectively. Collision energy was $20 \mathrm{~V}$ and $40 \mathrm{~V}$, and the scanning range of the mass analyzer was $m / z 50-1000$.

2.7. MS Data Processing Analysis. Data collection and procession were performed using the following software and databases: Agilent Mass Hunter Workstation software (version B.04.00) and Agilent MSC software (version B.07.00). Metabolites were identified through precise molecular weight, retention time, MS/MS fragmentation, the Metlin database (http://metlin.scripps.edu/index.php), and HMDB database (https://hmdb.ca/). The precursor ion mass tolerance was set to $10 \mathrm{ppm}$.

All data were normalized using the sum method. Subsequently, multivariate data analysis was conducted using the SIMCA-P (V 14.1, Umetrics, Sweden), including principal component analysis (PCA), partial least squares discriminant analysis (PLS-DA), and orthogonal partial least squares discriminant analysis (OPLS-DA). Permutation test was used to evaluate whether the established model was overfitting. A cross-validated analysis of variance was used to evaluate the reliability of the OPLS-DA model.

2.8. Statistical Analysis. All data were expressed as mean\pm standard deviation, and the data were analyzed using GraphPad Prism version 8.01 (GraphPad Software Inc., La Jolla, CA, USA). Student's $t$-test was used to assign significance $(p<0.05)$.

\section{Results}

3.1. Clinical Observation after Treatment of PMPE. Compared with the $\mathrm{BC}$ group, the weekly growth of the body weight in PMPE-treated rats significantly reduced (Figure 1(a)), whereas the liver index of PMPE-treated rats significantly increased $(p<0.05)$ (Figure $1(\mathrm{~b}))$. Liver pathology revealed liver cell degeneration, necrosis, and inflammatory cell infiltration after administration PMPE (Figure 1(c)).

3.2. Hepatotoxicity Assessment of the PMPE. Compared with the BC group, the levels of ALP, TAB, and TBIL increased significantly $(p<0.05)$, and the content of ALT, TG, and AST also showed increasing tendency (Figure 2).

\subsection{Metabolomic Profile Analysis of PMPE-Induced Liver} Injury. A total of 1827 and 992 features in plasma were extracted in the positive and negative ion mode, respectively. A representative plasma total ion current (TIC) chromatogram of the blank control group and the PMPE group are shown in Supplementary Figure S1.

3.4. Precision and System Stability Analysis. In order to estimate the precision and stability of the analytical method, QC samples were included into the entire analysis process, and the peak areas of the 6 characteristic ions were selected as evaluation indicators. As shown in Supplementary Table S1, the relative standard deviation (RSD) values of the peak area of the six characteristic ions of the QC samples were all less than $10 \%$.

3.5. Identification of Potential Liver Injury Biomarker and Pathway Analysis. PCA was used for visualizing group clustering and visualizing possible outliers. The BC group and PMPE group were obviously separated in the positive and negative ion mode (positive: $R^{2}=0.433, Q^{2}=0.177$; negative: $R^{2}=0.559, Q^{2}=0.344$ ), indicating that there was a significant difference between the $\mathrm{BC}$ and the PMPE groups (Figure 3). PLS-DA and permutation analysis were performed to evaluate the reliability of the model (positive: $R^{2}=0.487, Q^{2}=0.96$; negative: $R^{2}=0.605, Q^{2}=0.99$ ). The model validation of PLS-DA was performed at 200 permutations analysis (Supplementary Figure S1), and the results indicated that the PLS-DA model was credible and had no overfitting. OPLS-DA was carried out to reveal the differences of metabolites among groups as a supervised classification analysis method (positive: $R^{2}=0.568$, $Q^{2}=0.952$; negative: $R^{2}=0.605, Q^{2}=0.987$ ). Scatter plots and S-plot are shown in Figure 4. Variable important in projection (VIP) was used to evaluate the strength and explanatory power of the expression pattern of metabolites on the classification and discrimination of each group of samples. Differential metabolites were screened according to the VIP (VIP $>1.0)$ and $P$ values of Student's $t$-test $(p<0.05)$. A total of 342 differential characteristic ions from positive and negative ion patterns were selected. Based on the exact molecular ion mass, retention time of reference compounds, and metabolites database (METLIN, LIPID MAPS, and HMDB database), a total of 39 metabolites were identified as biomarker candidates of PMPE-induced liver injury finally. All candidate biomarkers were presented in Supplementary Table S2. 


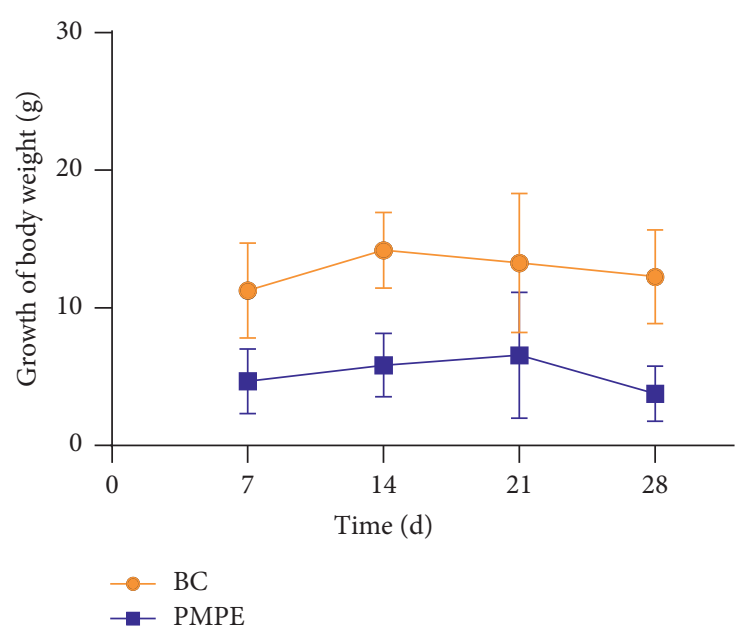

(a)

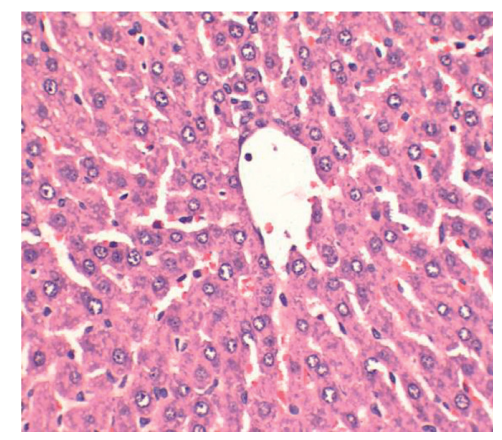

$\mathrm{BC}$

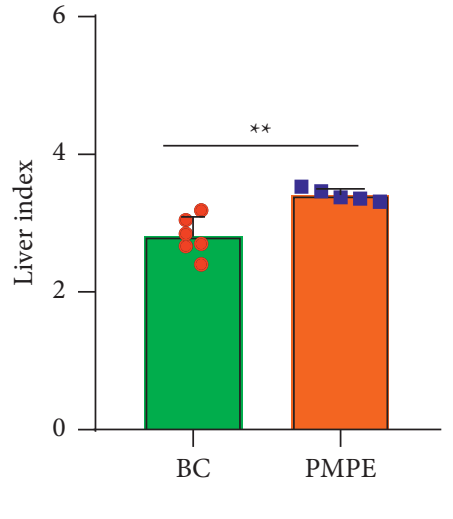

(b)

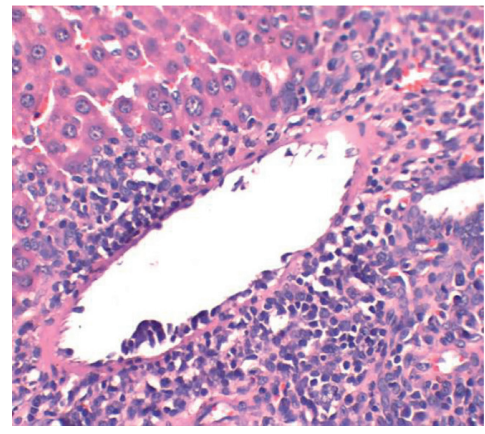

PMPE

(c)

Figure 1: The weight curve within 28 days of rats (a), liver index (b), and (c) Pathological sections of liver of rats after 28 days of administration of $60 \%$ ethanol extract of PMP (400 times under light microscope) in Blank Control (BC) and $60 \%$ ethanol extract of PMP (PMPE) groups ${ }^{*} p<0.05$, compared with the control group, ${ }^{* *} p<0.01$, compared with the control group.

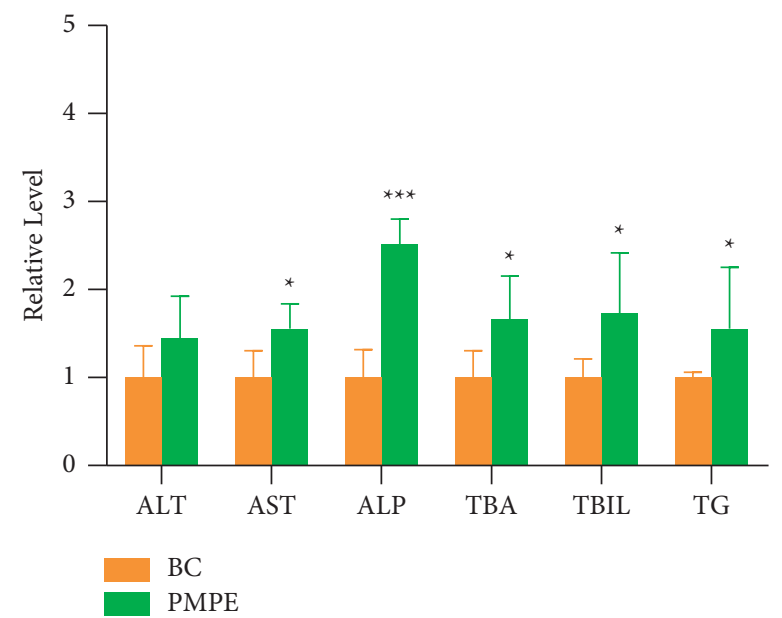

Figure 2: Serum relative levels of ALT, AST, ALP, TG, TBA, and TBIL in BC and PMPE groups, ${ }^{*} p<0.05,{ }^{* * *} p<0.0001$ compared with the control group. ALT, alanine aminotransferase; AST, aspartate aminotransferase; ALP, alkaline phosphatase; TG, triglyceride; TBA, total bile acid; TBIL total bilirubin. 


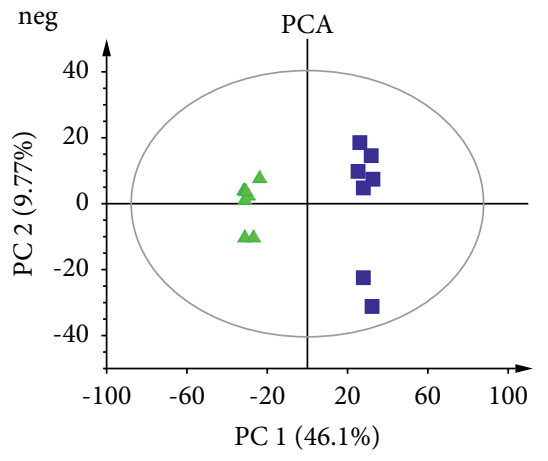

$\triangle$ PMPE

- BC

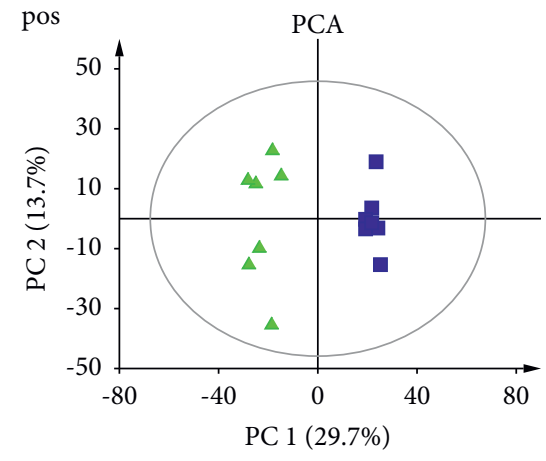

$\triangle \mathrm{PMPE}$

- $\mathrm{BC}$

(a)

(b)

Figure 3: PCA score plots of the LC-MS spectra from plasma. Negative (left) and Positive (right).

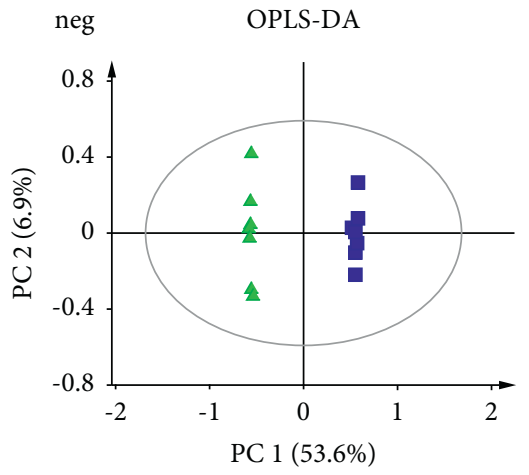

$\triangle$ PMPE

口C

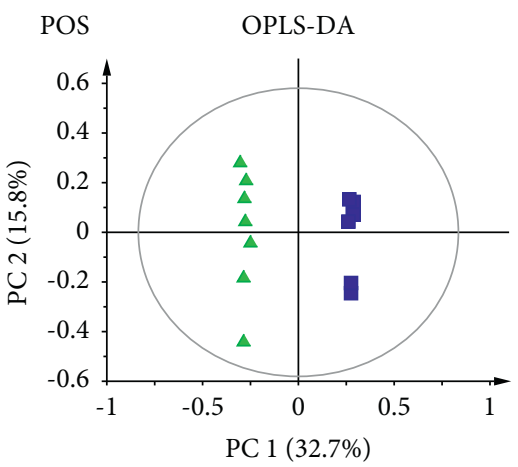

$\triangle$ PMPE

$\mathrm{BC}$

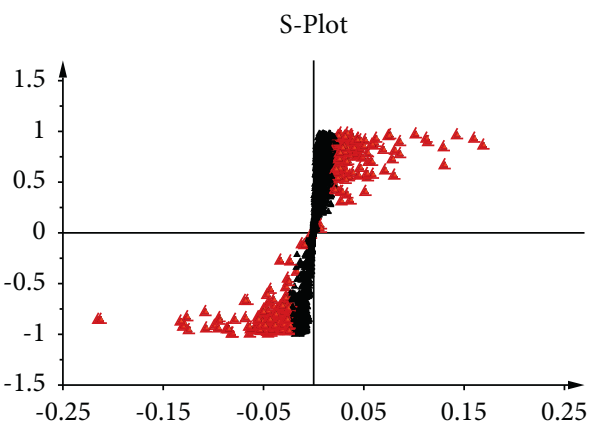

(a)

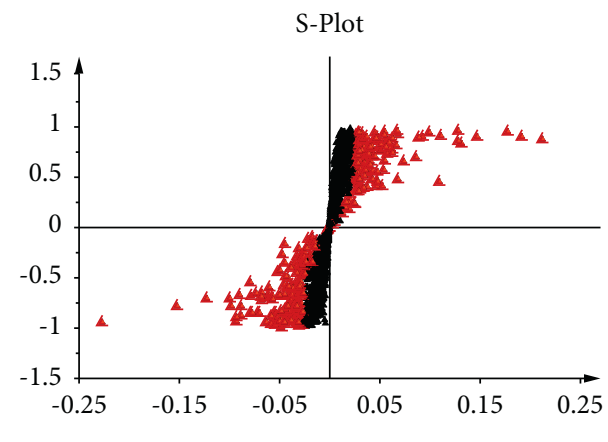

(b)

Figure 4: OPLS-DA analysis (left) and S-Plot (right) of the rat plasma from LC-MS spectra negative mode and positive mode.

The ROC curves were performed for the analysis of sorting out the potential diagnostic metabolites with high specificity and sensitivity to distinguish between the $\mathrm{BC}$ and PMPE groups. The diagnostic values were assessed by the area under the ROC curve (AUC), which was carried out using GraphPad Prism (version 8.0 San Diego, USA). The
AUCs of candidate liver injury biomarkers which were higher than 0.8 were regarded as potential biomarkers (Figure 5 and Supplementary Table S3). The results manifested that these biomarkers had better discrimination ability for healthy rats and liver injury rats. A total of 16 potential biomarkers were reconfirmed from the positive 


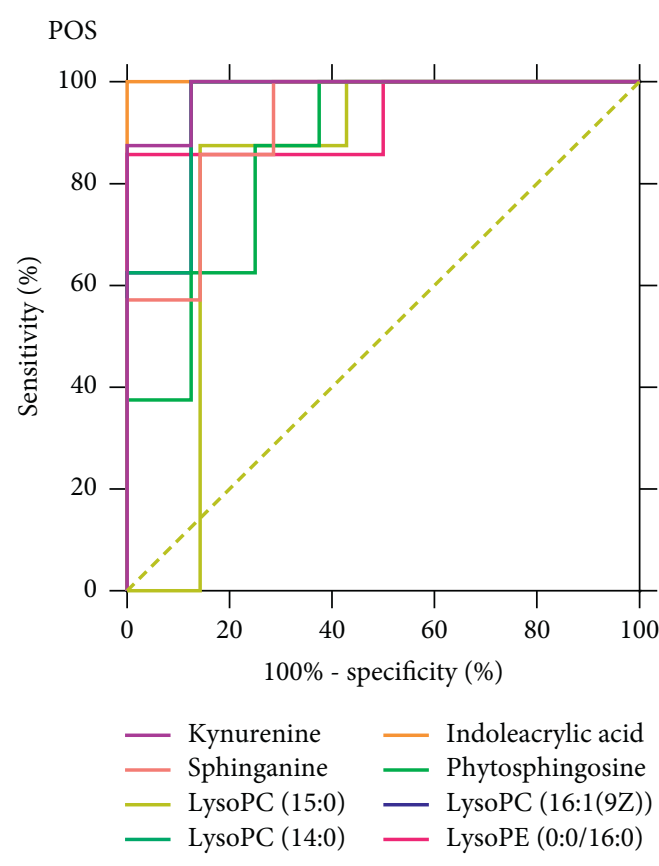

(a)

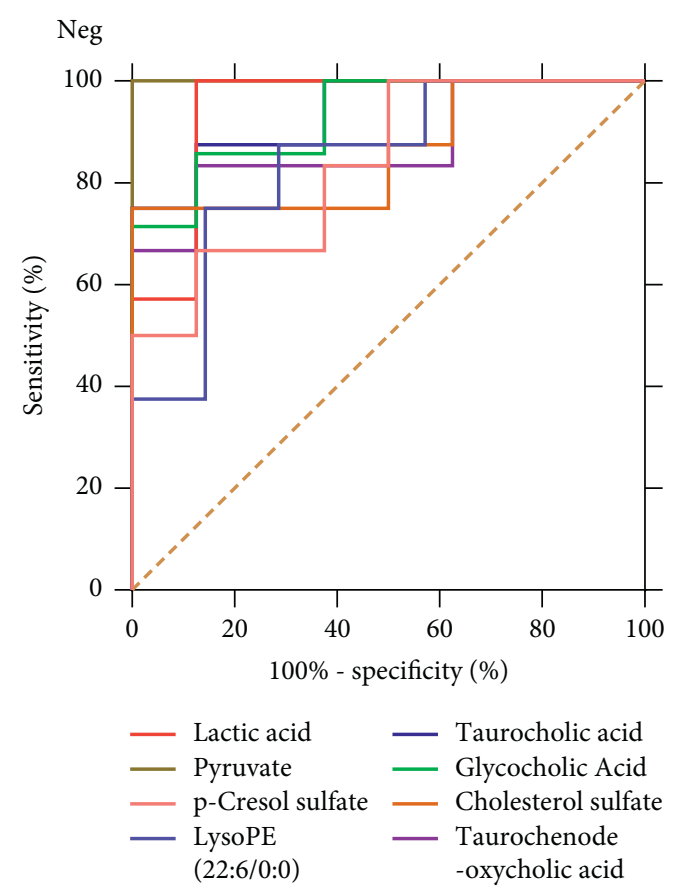

(b)

FIGURe 5: Potential biomarker ROC curve analysis. Positive (left) and Negative (right).

and negative ion patterns in rat plasma ( 8 for negative and 8 for positive). Potential biomarkers are summarized in Table 1. Six metabolites were significantly decreased in the PMPE group compared with those in the BC group, including kynurenine, lactic acid, pyruvate, sphinganine, phytosphingosine, and indoleacrylic acid (Figure 6).

To further elucidate the metabolic pathways of specific metabolites associated with PMPE-induced liver injury, we used the MetaboAnalyst4.0 (https://www.metaboanalyst.ca/ ) for enrichment and topological analysis of the KEGG signaling pathway. The most affected metabolic pathways were primary bile acid biosynthesis, sphingolipid metabolism, energy metabolism, glycerophospholipid metabolism, and tryptophan metabolism (Figure 7 and Supplementary Figure S2). In order to exhibit the results clearly, we constructed a network of potential metabolic pathways based on biomarkers in rat plasma (Figure 8).

\section{Discussion}

Biliary cholestasis and bile duct injury are the main clinical manifestations of cholestasis caused by drugs. Serum biochemical parameters ALP is preferred in clinical diagnosis. According to the above results, the levels of ALP, TBA, and TBIL showed significantly increased accompanied by liver cell degeneration, necrosis, and inflammatory cell infiltration, indicating that the PMPEinduced hepatotoxicity may be related to cholestasis. Meanwhile, the levels of taurochenodeoxycholic acid, taurocholic acid, and glycocholic acid were significantly increased in plasma of hepatotoxicity rats. Pathway enrichment and topology analysis also indicated that the biosynthesis pathway of bile acids was disrupted or interfered. In the process of generation, formation, transportation, and discharge of bile acid, any failure in those steps could cause cholestasis. Bile acid, especially hydrophobic toxic bile acids accumulation in liver cells, could induce inflammatory reactions by damaging mitochondria and lead to endoplasmic reticulum stress. These changes could directly destroy membranes of cell and organelle by virtue of detergent action and lead to hepatocyte injury finally. When hepatocytes are damaged, bile acid metabolism is impaired and reversely flows into blood $[18,19]$. These explain the mechanisms of serum TBA increased in rat with PMPE-induced liver injury.

As the endogenous ligand of FXR (farnesoid X receptor), bile acid is not only an important substance in lipid digestion and absorption but also serves as a signal molecule to transmit information through FXR-mediated signaling pathways and participate in the regulation of lipid metabolism in the body. FXR could monitor the metabolism of triglycerides and fatty acids through a variety of mechanisms. Generally, when FXR was activated by bile acids, SREPB-1c (sterol regulatory element-binding protein-1c), which is a major transcription factor involved in fat synthesis genes, was downregulated by SHP (recombinant small heterodimer partner) signal pathway in order to inhibit lipid synthesis. The activity of LDLR (low-density lipoprotein receptor) and VLDLR (very low-density lipoprotein receptor) was upregulated to increase lipid oxidation consumption and reduced lipid accumulation in the liver thereby [20]. Thus, lipids will accumulate in the liver when the activity of FXR was downregulated in the condition of disordered intrahepatic bile acid metabolism. 
TABLE 1: Biomarker candidates of PMPE-induced liver injury.

\begin{tabular}{|c|c|c|c|c|c|c|c|c|}
\hline & VIP & $\mathrm{FC}$ & $\mathrm{m} / \mathrm{z}$ & Formula & Biomaker & HMDB & KEGG & $P$-value \\
\hline \multirow{8}{*}{ Pos } & 4.98 & 0.72 & 188.06905 & $\mathrm{C}_{11} \mathrm{H}_{9} \mathrm{NO}_{2}$ & Indoleacrylic acid & HMDB00734 & - & $5.62 \mathrm{E}-05$ \\
\hline & 1.99 & 0.47 & 209.09043 & $\mathrm{C}_{10} \mathrm{H}_{12} \mathrm{~N}_{2} \mathrm{O}_{3}$ & Kynurenine* & HMDB0000684 & C00328 & $2.84 \mathrm{E}-04$ \\
\hline & 1.41 & 0.79 & 302.30336 & $\mathrm{C}_{18} \mathrm{H}_{39} \mathrm{NO}_{2}$ & Sphinganine & HMDB0000269 & C00836 & $4.11 \mathrm{E}-02$ \\
\hline & 1.62 & 0.76 & 318.29862 & $\mathrm{C}_{18} \mathrm{H}_{39} \mathrm{NO}_{3}$ & Phytosphingosine & HMDB0004610 & C12144 & $3.47 \mathrm{E}-02$ \\
\hline & 1.62 & 1.82 & 454.29073 & $\mathrm{C}_{21} \mathrm{H}_{44} \mathrm{NO}_{7} \mathrm{P}$ & LysoPE $(0: 0 / 16: 0)$ & HMDB0011473 & - & $1.10 \mathrm{E}-02$ \\
\hline & 2.62 & 2.58 & 468.30649 & $\mathrm{C}_{22} \mathrm{H}_{47} \mathrm{NO}_{7} \mathrm{P}$ & LysoPC(14:0) & HMDB0010379 & C04230 & $2.33 \mathrm{E}-03$ \\
\hline & 1.07 & 1.65 & 482.32192 & $\mathrm{C}_{23} \mathrm{H}_{48} \mathrm{NO}_{7} \mathrm{P}$ & LysoPC(15:0) & HMDB0010381 & $\mathrm{C} 04230$ & $4.79 \mathrm{E}-03$ \\
\hline & 3.74 & 2.35 & 494.32224 & $\mathrm{C}_{24} \mathrm{H}_{49} \mathrm{NO}_{7} \mathrm{P}$ & LysoPC $(16: 1(9 \mathrm{Z}))$ & HMDB0010383 & $\mathrm{C} 04230$ & $4.65 \mathrm{E}-02$ \\
\hline \multirow{8}{*}{ Neg } & 1.39 & 0.30 & 87.00888 & $\mathrm{C}_{3} \mathrm{H}_{4} \mathrm{O}_{3}$ & Pyruvate* & HMDB0000243 & $\mathrm{C} 00022$ & $3.10 \mathrm{E}-07$ \\
\hline & 5.7 & 0.47 & 89.02465 & $\mathrm{C}_{3} \mathrm{H}_{6} \mathrm{O}_{3}$ & Lactic acid & HMDB0000190 & C00256 & $1.02 \mathrm{E}-02$ \\
\hline & 2.49 & 1.54 & 187.00644 & $\mathrm{C}_{7} \mathrm{H}_{8} \mathrm{O}_{4} \mathrm{~S}$ & p-Cresol sulfate & HMDB11635 & C06677 & $4.23 \mathrm{E}-02$ \\
\hline & 2.05 & 2.38 & 464.30076 & $\mathrm{C}_{26} \mathrm{H}_{43} \mathrm{NO}_{6}$ & Glycocholic acid & HMDB0000138 & C01921 & $1.22 \mathrm{E}-02$ \\
\hline & 1.14 & 2.30 & 465.30223 & $\mathrm{C}_{27} \mathrm{H}_{46} \mathrm{O}_{4} \mathrm{~S}$ & Cholesterol sulfate & HMDB0000653 & C18043 & $1.42 \mathrm{E}-02$ \\
\hline & 2.33 & 1.80 & 496.27305 & $\mathrm{C}_{26} \mathrm{H}_{45} \mathrm{NO}_{7} \mathrm{~S}$ & Taurocholic acid & HMDB0000036 & $\mathrm{C} 05122$ & $3.02 \mathrm{E}-02$ \\
\hline & 3.8 & 2.23 & 498.28706 & $\mathrm{C}_{26} \mathrm{H}_{45} \mathrm{NO}_{6} \mathrm{~S}$ & Taurochenodeoxycholic acid & HMDB0000951 & C05465 & $3.30 \mathrm{E}-02$ \\
\hline & 1.05 & 1.50 & 524.27794 & $\mathrm{C}_{27} \mathrm{H}_{44} \mathrm{NO}_{7} \mathrm{P}$ & LysoPE $(22: 6 / 0: 0)$ & HMDB0011526 & - & $6.14 \mathrm{E}-03$ \\
\hline
\end{tabular}

Pos: positive; Neg: negative; FC: fold change; HMDB: human metabolome database; KEGG: Kyoto encyclopedia of genes and genomes. ${ }^{*}$ : compared with the reference standards.
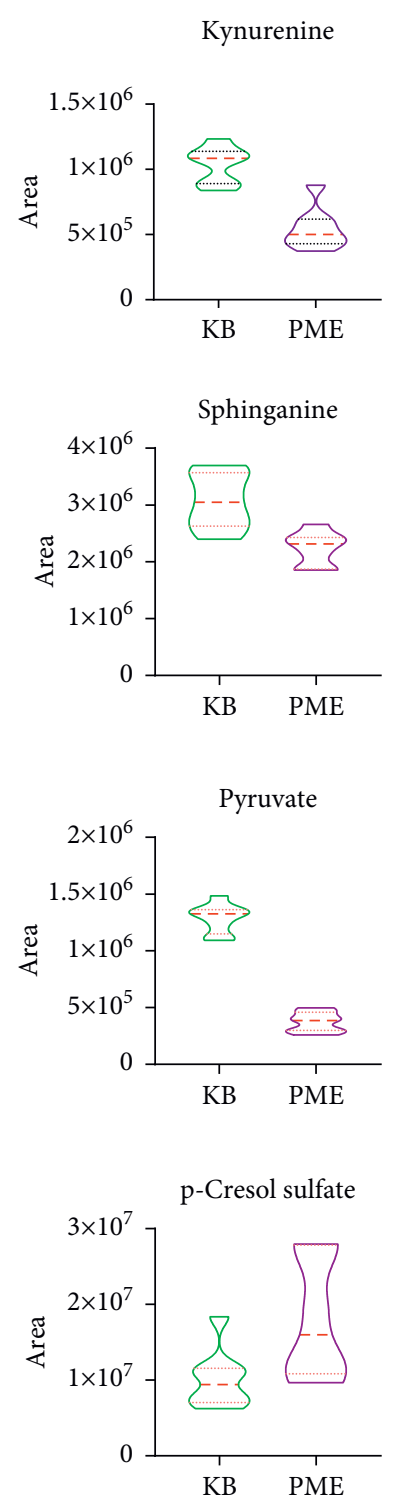

Taurochenodeoxycholic acid

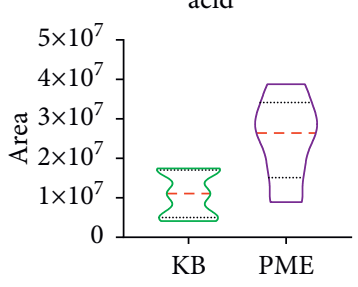

LysoPE (22:6/0:0)

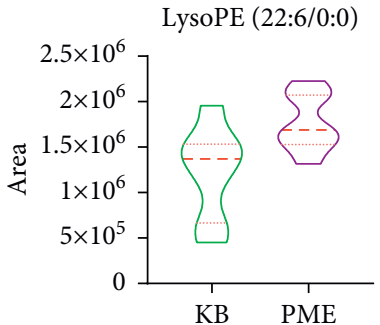

LysoPC (15:0)
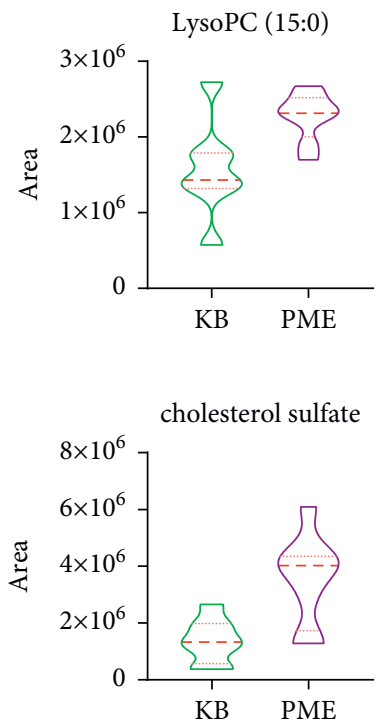

Phytosphingosine
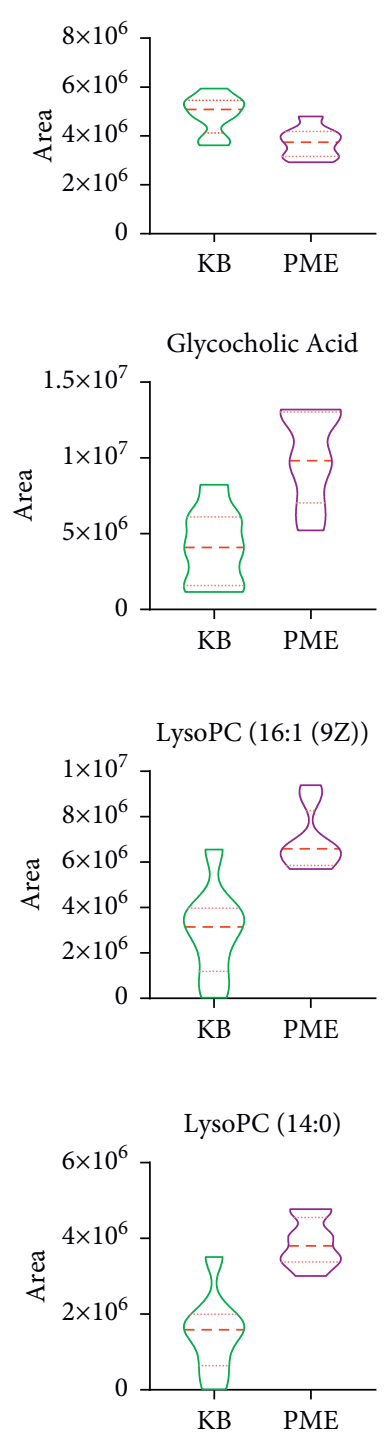
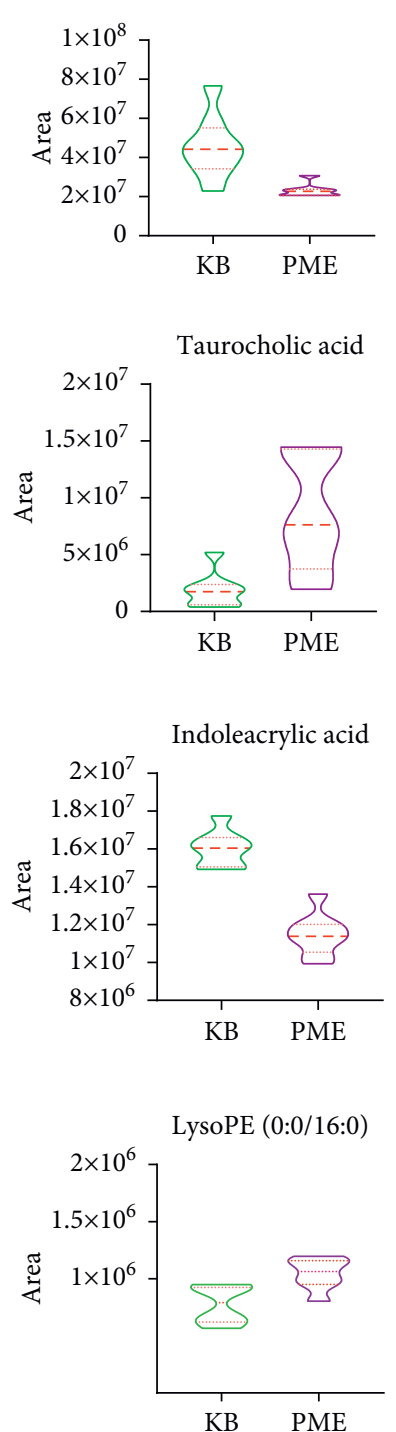

KB PME

Figure 6: The relative level of potential biomarker content. 
Metabolite Sets Enrichment Overview

Primary bile acid biosynthesis

Sphingolipid metabolism

Pyruvate metabolism

Glycolysis/Gluconeogenesis

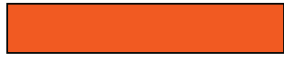

$P$ value

Tryptophan metabolism

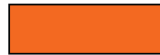

Taurine and hypotaurine metabolism

Citrate cycle (TCA cycle)

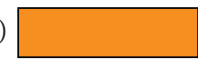

$3 e-01$

Alanine, aspartate and glutamate metabolism

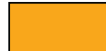

Gloxylate and dicarboxylate metabolism

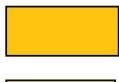

Glycine, serine and threonine metabolism

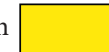

$6 e-01$

Cysteine and methionine metabolism

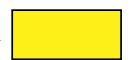

Arginine and proline metabolism

Tyrosine metabolism

Steroid hormone biosynthesis

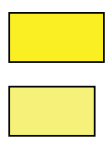

is

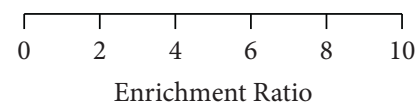

Figure 7: Differential metabolites pathway enrichment analysis of PMPE-induced liver injured.

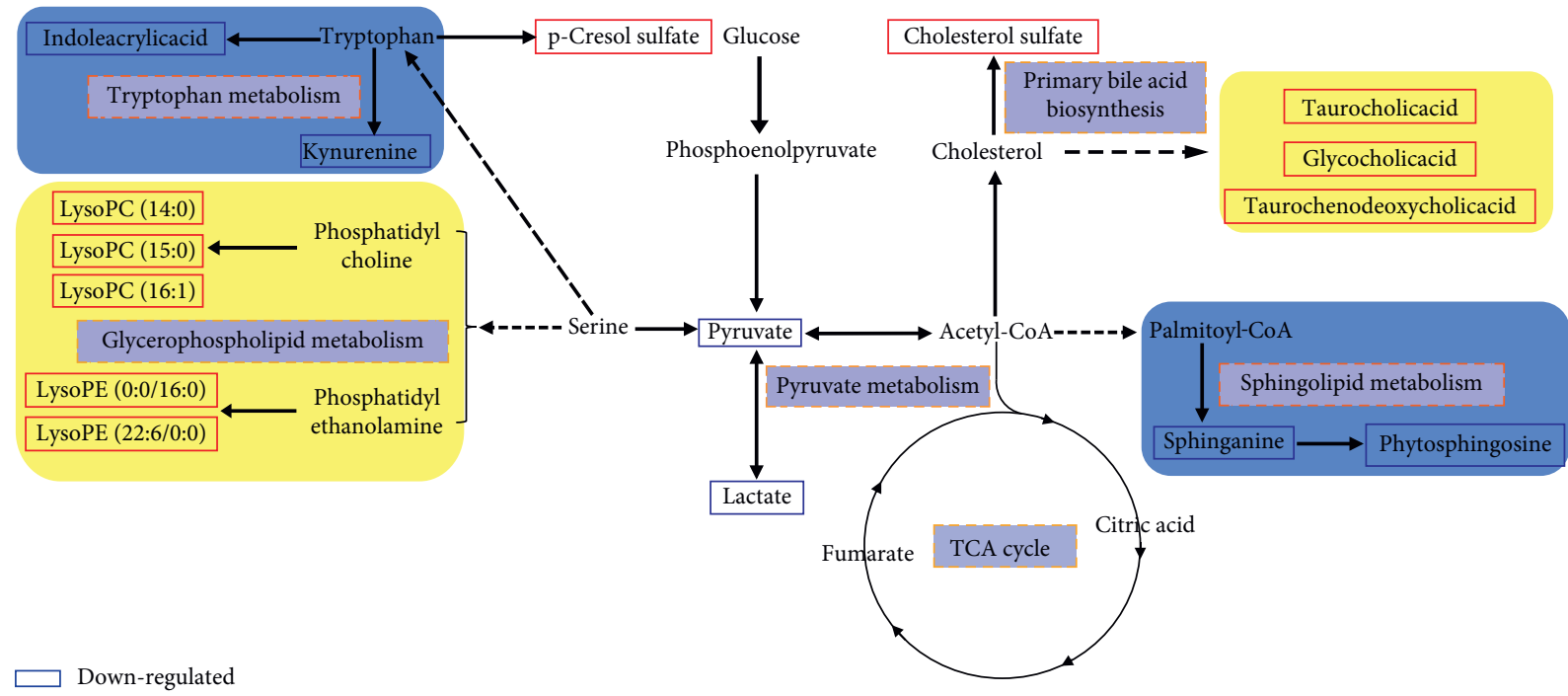

$\square$ Up-regulated

Metabolism pathway

Figure 8: Potential metabolic pathway of PME-induced liver injured. 
Phosphatidylcholine (PC) and phosphatidylethanolamine (PE), two most affluent glycerophospholipids, play a pivotal biological function in regulating lipoprotein metabolism [21]. They can be metabolized by phospholipase A2 (PLA2) into LPC (lysophosphatidylcholine), LPE (lysophosphatidylethanolamine), and arachidonic acid, which plays a very important role in lipid inflammation [22]. Metabolic disorders of these lipids result in changes in membrane lipid composition and affect the physical properties and functional integrity of the membrane. These could lead to hepatocellular apoptosis, inflammation, and the progression of liver disease. In addition, studies have reported that LPC has a certain proinflammatory function and is a new type of inflammatory lipids [23]. The experimental studies in vivo have shown that LPC can induce hepatitis which is harmful to the liver. It can also induce hepatocyte lipid apoptosis by activating GPCRs (G proteincoupled receptors) and depolarization of mitochondrial membrane [24]. Many researches have shown that the incubation of primary hepatocytes with LPC could induce cell death by apoptosis [25]. In our study, LPC and LPE were the most increased lipids in the oral administration group of PME, and the accumulation of these metabolites caused hepatocyte dysfunction. Sphingolipids are an important ingredient of the biofilm structure. As the important active molecules in organisms, sphingolipids and their metabolites have been identified as new biomarkers of chronic hepatopathy disease and hepatocellular carcinoma (HCC) [26].

As the site of gluconeogenesis and glycogen synthesis, the liver plays an important role in regulating blood sugar levels. Bile acids could regulate glucose metabolism through FXR-SHP or bile acid-TGR5 (Takeda G proteincoupled receptor 5) signal pathway [27]. Some representative important metabolites related to energy metabolism and tryptophan metabolism were detected in this study, such as pyruvic acid, lactic acid, kynurenine, and indoleacrylic acid. These metabolites were significantly reduced. The low level of kynurenine is closely related to abnormal liver function and energy metabolism. It has been reported that drug-induced liver injury is related to the mitochondrial function and energy homeostasis [28]. Moreover, the decrease of indoleacrylic acid is related to the disorder of tryptophan metabolism in the liver. The parallels with our findings are striking. These results indicated that liver injury affected the disorder of these metabolic pathways. In conclusion, all results indicated that PMP affected metabolic disorder of bile acids, lipids, and glucose metabolism; impaired liver function; and could induce liver injury finally.

Compared with the control group, the content of p-cresol sulfate in the PME group was significantly increased. Cholesterol sulfate is a representative substance of uremic toxin produced by tyrosine. The results suggested that the kidney may be damaged in different degrees, which may be attributed to the aggravation of kidney metabolism after liver injury. In addition, the disorder of bilirubin metabolism and blood circulation could also lead to renal function decline (hepatorenal syndrome) [29].

\section{Conclusion}

In summary, LC-MS-based plasma metabolomics analysis combined with ROC curve analysis method provides an integrated view of the metabolic features of PMP-induced hepatoxicity. The differential metabolites were identified. Metabolic pathway analysis indicated that the bile acid biosynthesis pathway was the main pathways affected by PMP, which may be the cause of liver injury. The sphingolipid metabolism, energy metabolism, glycerophospholipid metabolism, and tryptophan metabolism pathway were also involved. These discriminating metabolites may help to understand the pathogenesis of liver injury and provide a good prospect for the clinical diagnosis of PMPE-induced liver injury.

\section{Abbreviations}

PMP: $\quad$ Polygonum Multiflorum Radix Preparata

PM: $\quad$ Polygoni Multiflori

PMPE: $\quad 60 \%$ ethanol extract of PMP

ROC: $\quad$ Receiver operating characteristic

UHPLC-Q- Ultrahigh performance liquid

TOF-MS: chromatography coupled with quadrupole

QC: Quality control

RSD: $\quad$ Relative standard deviation.

\section{Data Availability}

Data are available from the corresponding author upon request.

\section{Conflicts of Interest}

The authors declare that they have no conflicts of interest.

\section{Authors' Contributions}

Liming Wang and Zhida Wang contributed equally to this work. They performed the experiment and wrote the manuscript. Yanchao Xing analyzed the data. Erwei Liu and Xiumei Gao revised the manuscript. Manuscript editing and review were performed by Linlin Wang and Zhifei Fu. All authors read and approved the final manuscript.

\section{Acknowledgments}

This work was supported by the Innovation Team and Talents Cultivation Program of National Administration of Traditional Chinese Medicine (ZYYCXTD-C-202009), National Key R\&D Program of China (2018YFC1704500), Important Drug Development Fund, Ministry of Science and Technology of China (2019ZX09201005-002-007), Science and Technology Program of Tianjin, China (19ZYPTJC00060), and Education Commission Research Program of Tianjin, China (2019KJ079).

\section{Supplementary Materials}

Table S1: the relative standard deviation (RSD) values of the peak area of the six characteristic ions of the QC samples. 
Table S2: biomarker candidates of PMPE-induced liver injury in positive and negative mode. Table S3: the AUC values of candidate liver injury biomarkers. Figure S1: TIC of the LC-MS spectra, PLS-DA analysis, and permutations analysis in negative mode and positive mode. Figure S2: analysis of metabolic pathways related to liver injury. (Supplementary Materials)

\section{References}

[1] H. Yu, L. Wang, Y. He et al., "Advances in the study of the potential hepatotoxic components and mechanism of polygonum multiflorum," Evidence-based Complementary and Alternative Medicine, vol. 2020, Article ID 6489648, 12 pages, 2020.

[2] China Pharmacopoeia Committee, Pharmacopoeia of the People's Republic of China, China Pharmacopoeia Committee, Beijing, China, 2020.

[3] L. Lin, B. Ni, H. Lin et al., "Traditional usages, botany, phytochemistry, pharmacology and toxicology of Polygonum multiflorum Thunb.: a review," Journal of Ethnopharmacology, vol. 159, pp. 158-183, 2015.

[4] T. Teka, L. Wang, J. Gao et al., "Polygonum multiflorum: recent updates on newly isolated compounds, potential hepatotoxic compounds and their mechanisms," Journal of Ethnopharmacology, vol. 271, Article ID 113864, 2021.

[5] J. Y. Shin, Y.-H. Choi, and J. Kim, "Polygonum multiflorum extract support hair growth by elongating anagen phase and abrogating the effect of androgen in cultured human dermal papilla cells," BMC Complementary Medecine Therapies, vol. 20, no. 1, p. 144, 2020.

[6] M. Han, J. Lu, G. Zhang et al., "Mechanistic studies on the use of polygonum multiflorum for the treatment of hair graying," BioMed Research International, vol. 2015, Article ID 651048, 8 pages, 2015.

[7] H. Wang and X. Li, "Clinical analysis of 33 cases of drug induced liver injury caused by Polygonum multiflorum Thunb and its preparations," Chinese Journal of Integrated Traditional and Western Medicine on Liver Diseases, vol. 28, no. 1, pp. 25-27, 2018.

[8] Y. Zhu, Y. Li, Y. Wang et al., "Analysis of clinical characteristics in 595 patients with Herb-induced liver injury," Chinese Journal of Integrated Traditional and Western Medicine, vol. 36, no. 1, pp. 44-48, 2016.

[9] J. Hoofnagle, J. Serrano, and J. Knoben, "LiverTox: a website on drug-induced liver injury," Hepatology, vol. 57, no. 3, pp. 873-874, 2013.

[10] National Medical Products Administration, ZTZL-201410354. 2014 -07-16, National Medical Products Administration, Beijing, China, 2014.

[11] A. C. Schrimpe-Rutledge, S. G. Codreanu, S. D. Sherrod, and J. A. McLean, "Untargeted metabolomics strategies-challenges and emerging directions," Journal of the American Society for Mass Spectrometry, vol. 27, no. 12, pp. 1897-1905, 2016.

[12] F. R. Pinu, D. J. Beale, A. M. Paten et al., "Systems biology and multi-omics integration: viewpoints from the metabolomics research community," Metabolites, vol. 9, no. 4, p. 76, 2019.

[13] S. Forcisi, F. Moritz, B. Kanawati et al., "Liquid chromatography-mass spectrometry in metabolomics research: mass analyzers in ultra high pressure liquid chromatography coupling," Journal of Chromatography A, vol. 1292, pp. 51-65, 2013.
[14] L. Liang, J. Xu, W. Zhou et al., "Integrating targeted and untargeted metabolomics to investigate the processing chemistry of Polygoni Multiflori Radix," Frontiers in Pharmacology, vol. 9, p. 934, 2018.

[15] Y. Huang, X. Zhao, Z. Zhang, S. Chen, S. Li, and Z. Shi, "Metabolomics profiling and diagnosis biomarkers searching for drug-induced liver injury implicated to polygonum multiflorum: a cross-sectional cohort study," Frontiers in Medecine, vol. 7, Article ID 592434, 2020.

[16] L. Wang, M. Sang, E. Liu et al., "Rapid profiling and pharmacokinetic studies of major compounds in crude extract from Polygonum multiflorum by UHPLC-Q-TOF-MS and UPLC-MS/MS," Journal of Pharmaceutical and Biomedical Analysis, vol. 140, pp. 45-61, 2017.

[17] Y. Xing, L. Wang, C. Wang et al., "Pharmacokinetic studies unveiled the drug-drug interaction between trans-2,3,5,4'tetrahydroxystilbene- 2 -O- $\beta$-d-glucopyranoside and emodin that may contribute to the idiosyncratic hepatotoxicity of Polygoni Multiflori Radix," Journal of Pharmaceutical and Biomedical Analysis, vol. 164, pp. 672-680, 2019.

[18] Q. Dong, N. Li, Q. Li et al., "Screening for biomarkers of liver injury induced by Polygonum multiflorum: a targeted metabolomic study," Frontiers in Pharmacology, vol. 6, p. 217, 2015.

[19] D. Sokolovic, J. Nikolic, G. Kocic, T. Jevtovic-Stoimenov et al., "The effect of ursodeoxycholic acid on oxidative stress level and DNase activity in rat liver after bile duct ligation," Drug and Chemical Toxicology, vol. 36, no. 2, pp. 141-148, 2013.

[20] B. Goodwin, S. Jones, R. Price et al., "A regulatory cascade of the nuclear receptors FXR, SHP-1, and LRH-1 represses bile acid biosynthesis," Molecular Cell, vol. 6, no. 3, pp. 517-526, 2000.

[21] G. Drin, "Topological regulation of lipid balance in cells," Annual Review of Biochemistry, vol. 83, pp. 51-77, 2014.

[22] E. A. Dennis and P. C. Norris, "Eicosanoid storm in infection and inflammation," Nature Reviews Immunology, vol. 15, no. 8, pp. 511-523, 2015.

[23] S. Law, M. Chan, G. K. Marathe, F. Parveen, C. Chen, and L. Ke, "An updated review of lysophosphatidylcholine metabolism in human diseases," International Journal of Molecular Sciences, vol. 20, no. 5, p. 1149, 2019.

[24] V. B. O’Donnell, J. Rossjohn, and M. J. O. Wakelam, "Phospholipid signaling in innate immune cells," Journal of Clinical Investigation, vol. 128, no. 7, pp. 2670-2679, 2018.

[25] K. Kakisaka, S. Cazanave, C. Fingas et al., "Mechanisms of lysophosphatidylcholine-induced hepatocyte lipoapoptosis," American Journal of Physiology - Gastrointestinal and Liver Physiology, vol. 302, no. 1, pp. 77-84, 2012.

[26] A. D. Patterson, O. Maurhofer, D. Beyoğlu et al., "Aberrant lipid metabolism in hepatocellular carcinoma revealed by plasma metabolomics and lipid profiling," Cancer Research, vol. 71, no. 21, pp. 6590-6600, 2011.

[27] D. Duran-Sandoval, B. Cariou, F. Percevault et al., "The farnesoid X receptor modulates hepatic carbohydrate metabolism during the fasting-refeeding transition," Journal of Biological Chemistry, vol. 280, no. 33, pp. 29971-29979, 2005.

[28] M. Cuykx, C. Beirnaert, R. Rodrigues, K. Laukens, T. Vanhaecke, and A. Covaci, "Untargeted liquid chromatography-mass spectrometry metabolomics to assess druginduced cholestatic features in HepaRG ${ }^{\circledR}$ cells," Toxicology and Applied Pharmacology, vol. 379, Article ID 114666, 2019.

[29] Y. Yan, N. Shi, X. Han, G. Li, B. Wen, and J. Gao, "UPLC/MS/ MS-based metabolomics study of the hepatotoxicity and nephrotoxicity in rats induced by Polygonum multiflorum Thunb," ACS Omega, vol. 5, no. 18, pp. 10489-10500, 2020. 